\title{
FLEXIBLE PAYMENT PLANS FOR PATIENTS
}

Simplyhealth Professionals has announced the launch of a new flexible payment plan for both its member practices and non-members which will help patients to spread the cost of both dental and facial aesthetic treatments.

The new Flexible Payment Plans will make treatments more accessible and affordable for all patients as they can set the price and payment length with their dentist so it suits individual budgets. Patients can opt for treatments that they might have previously thought were unaffordable.

Dentists will agree with each patient how much they pay each month and how long their treatment will take. They can offer the patient an ongoing monthly plan for more regular treatments, or a choice of three to ten monthly payments for a one-off treatment, helping the patient to spread the cost of treatment and make it more affordable for them.

This is the first time that Simplyhealth Professionals has created a payment plan that

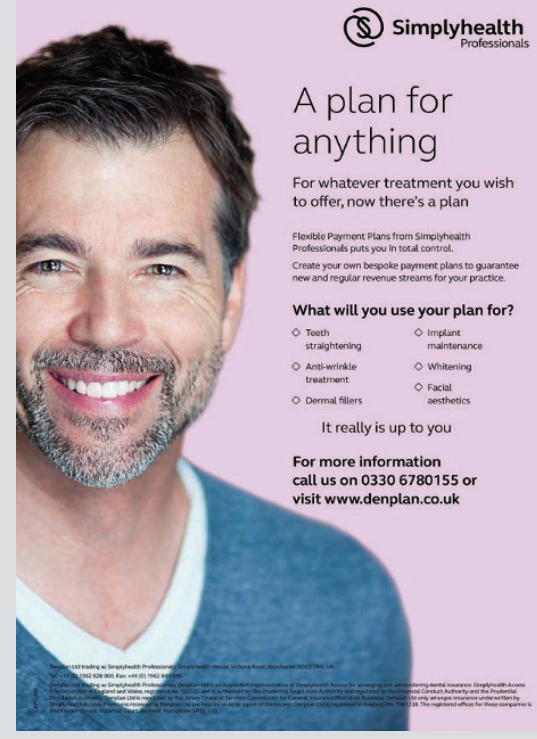

can be tailored to support dentists providing an increasingly diverse mix of cosmetic dental and facial aesthetic treatments in their practices. Flexible Payment Plans will encourage patients to opt for new or higher cost treatments which they previously might not have considered due to the price.

This is also the first time that the company has offered a payment plan for non-members. This will appeal to those dentists who specialise in high end treatments such as facial or cosmetic work who want a flexible plan to cover this treatment, in addition to traditional restorative and preventive dental treatments.

Flexible payment plans are particularly well suited for more costly treatments and nonroutine treatments such as tooth whitening and straightening, implant maintenance, facial aesthetics and cosmetic dentistry, or as a restorative treatment plan for crowns, bridges and dentures. It will open up greater choice for patients in non-dental treatments such as anti-wrinkle or dermal fillers.

Dentists can also create, brand and promote their own Hygiene or Whitening Plans using the Flexible Payment Plans platform.

Once the dentist has agreed the treatment plan and costs with their patient, they set up the plan on the Flexible Payment Plan portal. Once the patient's personal and payment details are entered, the plan is immediately set up and ready to start, and the patient can book appointments for their treatment. The dentist can access the portal at any time to check the status of an individual plan.

Practices can also use Simplyhealth Professionals' online design service to create bespoke posters and literature for individual flexible payment plans.

www.denplan.co.uk/dentists/flexiblepayment-plans

\section{A SUSTAINABLE, FISH-FRIENDLY TOOTHBRUSH}

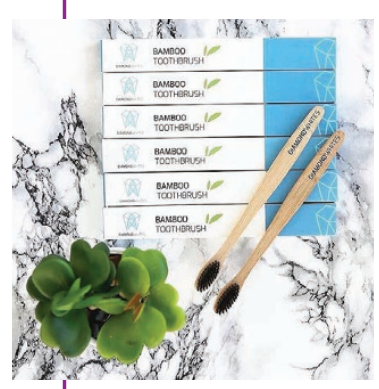

Diamond Whites

have launched their brand new Bamboo Toothbrush.

The Diamond

Whites Bamboo

Toothbrush is a biodegradable, eco-friendly

toothbrush that works in perfect harmony with Diamond Whites pastes and polishes to help keep teeth healthy and clean.

The BBC estimates that by 2050 there will be more plastic than fish in our oceans, and as dental professionals advise patients to replace their toothbrush every three months, why not advise them to switch to a sustainable toothbrush that's a friend to the environment as well as their teeth?

The Diamond Whites Bamboo

Toothbrush is available now for $£ 2.99$ at www.diamondwhites.co.uk.

\section{SCALING NEW HEIGHTS IN PATIENT CARE}

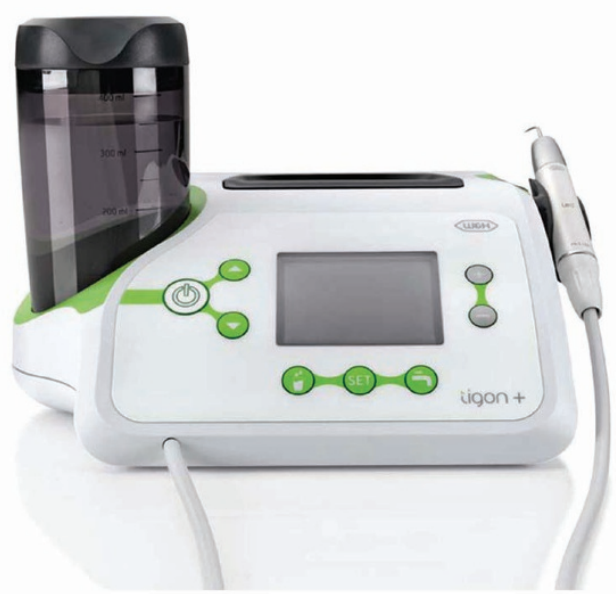

Regular dental cleaning can be particularly painful for periodontal patients that already have inflamed gums.

That's why W\&H has developed an ultrasonic treatment unit to make scaling teeth even easier for practitioners, and more comfortable for patients.

With five preset treatment programmes to choose from, the dynamic design of the Tigon+ means that it can be used in prophylaxis, periodontology, endodontics and restorations.

Thanks to temperature-controlled coolant, the Tigon+ treats patients to pleasantly warm water, meaning it can be used on even the most sensitive teeth and gums.

Three modes allow you to define the scaler power according to preference, so you can be sure that you are delivering quality treatment that is neither too gentle, nor too harsh.

What's more, the easy-to-operate display of the Tigon+ makes it an incredibly user-friendly device, so you can increase the efficacy of your practice.

Contact the expert team at W\&H to find out more about the benefits you can reap from the scaling power of the Tigon+.

To find out more visit www.wh.com/ en_uk, call 01727874990 or email office.uk@wh.com. 